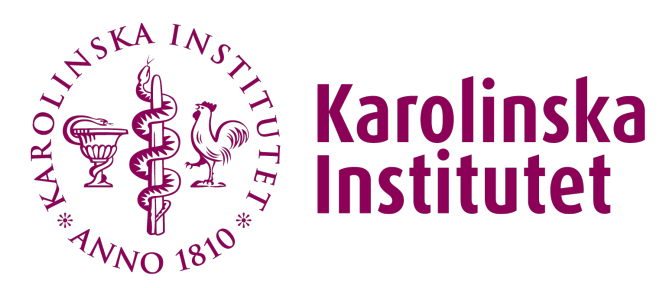

Karolinska Institutet

http://openarchive.ki.se

This is a Peer Reviewed Accepted version of the following article, accepted for publication in ACS Nano.

2019-04-24

\title{
Effects of design choices on the stiffness of wireframe DNA origami structures
}

Benson, Erik; Mohammed, Abdulmelik; Rayneau-Kirkhope, Daniel; Gådin, Andreas; Orponen, Pekka; Högberg, Björn

ACS Nano. 2018 Sep 25;12(9):9291-9299.

American Chemical Society

http://doi.org/10.1021/acsnano.8b04148

http://hdl.handle.net/10616/46757

If not otherwise stated by the Publisher's Terms and conditions, the manuscript is deposited under the terms of the Creative Commons Attribution-NonCommercial-NoDerivatives License (http://creativecommons.org/licenses/by-nc-nd/4.0/), which permits non-commercial re-use, distribution, and reproduction in any medium, provided the original work is properly cited, and is not altered, transformed, or built upon in any way. 


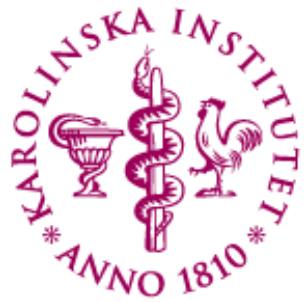 \\ Karolinska Institutet}

This is an author produced version of a paper published in ACS Nano

This paper has been peer-reviewed, but does not include the final publisher proof-corrections or journal pagination.

\section{Citation for the published paper:}

Benson E, Mohammed A, Rayneau-Kirkhope D, Gådin A, Orponen P, Högberg B. Effects of Design Choices on the Stiffness of Wireframe DNA Origami Structures. ACS Nano. 2018 Sep 25;12(9):9291-9299.

URL: http: //doi.org/10.1021/acsnano.8b04148

Access to the published version may require subscription.

Published with permission from:

ACS Publications, American Chemical Society 


\title{
Effects of Design Choices on the Stiffness of
}

\section{Wireframe DNA Origami Structures}

\author{
Erik Benson ${ }^{1}$, Abdulmelik Mohammed ${ }^{2}$, Daniel Rayneau-Kirkhope ${ }^{3,4}$, Andreas Gådin ${ }^{1}$, Pekka \\ Orponen $^{2 *}$, and Björn Högberg ${ }^{1 *}$
}

1. Department of Medical Biochemistry and Biophysics, Karolinska Institutet, SE-17177 Stockholm, Sweden.

2. Department of Computer Science, Aalto University, FI-00076 Aalto, Finland.

3. Aalto Science Institute, School of Science, Aalto University, FI-00076 Aalto, Finland.

4. Department of Applied Physics, Aalto University, FI-00076 Aalto, Finland.

KEYWORDS: DNA nanostructures, DNA origami, wireframe DNA origami, beam theory, rigidity, persistence length, oxDNA

ABSTRACT: DNA origami is a powerful method for the creation of 3D nanoscale objects, and in the past few years, interest in wireframe origami designs has increased due to their potential for biomedical applications. In DNA wireframe designs, the construction material is double stranded DNA, which has a persistence length of around $50 \mathrm{~nm}$. In this work, we study the effect of various design choices on the stiffness versus final size of nanoscale wireframe rods, given the constraints on origami designs set by the DNA origami scaffold size. An initial theoretical 
analysis predicts two competing mechanisms limiting rod stiffness, whose balancing results in an optimal edge length. For small edge lengths, the bending of the rod's overall frame geometry is the dominant factor, while the flexibility of individual DNA edges has a greater contribution at larger edge lengths. We evaluate our design choices through simulations and experiments and find that the stiffness of the structures increases with the number of sides in the cross section polygon, and that there are indications of an optimal member edge length. We also ascertain the effect of nicked DNA edges on the stiffness of the wireframe rods and demonstrate that ligation of the staple breakpoint nicks reduces the observed flexibility. Our simulations also indicate that the persistence length of wireframe DNA structures significantly decreases with increasing monovalent salt concentration.

The concept of structural DNA nanotechnology was first conceived in the early 1980 's ${ }^{1}$ and experimentally demonstrated in the following decade. ${ }^{2}$ Subsequently, various design approaches have been employed to create DNA nanostructures of increasing size and complexity. The introduction of the DNA origami technique in $2006^{3}$ significantly accelerated this development and functionalized DNA nanostructures, with potential applications in plasmonics, ${ }^{4,5}$ electronics, ${ }^{6,7}$ and cell signaling studies ${ }^{8,9}$ have since been demonstrated.

Analogous to other construction materials, there is a fundamental interest in how the properties of DNA translate to the mechanics of structures assembled from it. Already in his seminal 1982 paper, ${ }^{1}$ Seeman astutely observed the effect that the length-dependent stiffness of doublestranded DNA would have on the flexibility of DNA assemblies. In the following decades, many of the demonstrated structures, most notably those based on the DNA origami technique, ${ }^{3,10,11}$ 
relied on the parallel packing of DNA double helices. The mechanical properties of such structures have been studied through experiments, ${ }^{12,13}$ theoretical analyses ${ }^{14}$ and simulations. ${ }^{15-17}$

In parallel with packed origami designs, many wireframe designs with moderate edge-counts have also been demonstrated. ${ }^{18-24}$ Wireframe designs are appealing because their lower packing density permits their assembly at physiological salt concentrations, making them suitable for biomedical applications; they can also allow assembly of larger structures from a given length of scaffold strand. ${ }^{25}$ Latticework design is widely used in macroscopic construction as it potentially offers superior strength-to-weight ratio compared to solid beams. ${ }^{26,27}$ Utilizing the same design principles at the nanoscale may allow the assembly of large yet stiff DNA nanostructures. In this context, it had previously proved challenging to produce large DNA origami structures of mesh wireframes, as a manual routing of scaffold strands along the wireframes becomes infeasible for most large meshes. In 2015, we introduced an automated scaffold routing method, implemented in the BSCOR package, that enables the rapid generation of DNA origami designs of polyhedral mesh wireframes. ${ }^{28}$ Within the limits set by physical geometric constraints and algorithmic complexity, the method enables the synthesis of any wireframe of a polyhedral mesh inflatable to a sphere. Other approaches have also been demonstrated for the generation of wireframe DNA origami structures. ${ }^{29,30}$

In this paper, we investigate the effect of design choices on the stiffness of 3D DNA nanostructures assembled as polyhedral meshes. More concretely, we focus on rod designs, as they are a fundamental structural element of many larger assemblies and their overall stiffness can easily be estimated through their persistence length. ${ }^{31}$ We start by analyzing the properties of wireframe rods using beam theory, ${ }^{32}$ considering the stiffening due to geometric effects (specifically, increasing the second moment of area) while also analyzing the effect of increased 
uncertainty in end-to-end length of frame members. Moreover, we apply a combination of coarse-grained molecular dynamics simulations and wet-lab experiments to evaluate the effect of design choices on the stiffness of the assembled rods. For our study, we chose rod designs generated from triangulated meshes, where all internal vertices are incident to six edges. The pretriangulated meshes can be viewed as a line of axially stacked prismatic modules referred to here as frame unit cells (cf. Figures 1 and 2).

Here we look at several design parameters for a wireframe rod that may have crucial effects on its stiffness: 1) The number of sides in a rod cross section. 2) Global scaling of all edges and 3) Varying the aspect ratios of the constituent unit cells, or rod segments, by scaling the base size.

In the first parameter, the number of sides of the cross-section polygon, this can be varied from the simplest triangular cross-section with three sides to cross-section profiles with more sides. This however induces a tradeoff in the usage of DNA per unit rod length: since the number of edges in the wireframe structure grows linearly with respect to the number of sides of the crosssection polygon, a rod with a hexagonal cross-section will use approximately twice as much material as a rod with a triangular cross-section, given identical edge lengths.

Second, a global isotropic scaling of the edges can be applied. When rendering a mesh wireframe to a DNA nanostructure design, in the BSCOR package we use a scaling parameter that determines the number of base pairs used to render the mesh edges in the abstract design. Utilizing this parameter, rod designs of various lengths can easily be generated from the same mesh. A rod with a desired length can be composed from either many small-frame unit cells or fewer large-frame unit cells, and in this case, the designs with the larger modules will have larger 
cross sections. We adopt such a design set of structures with the same overall length to study the impact of isotropic scaling on optimizing the stiffness of DNA rods, noting that the designs scaled in this way should have little variation in material usage per unit length of wireframe rod.

And third, as an alternative to isotropic scaling, the transversal edges can also be scaled independently of the axial edges, altering only the areas of the cross-sections but not the lengths of the final designs. In such transversal or base scaling, the material use will increase with increasing cross-section area.

\section{Results/Discussion}

The effect of the different scaling parameters on the stiffness of the assembled structures is non-trivial. When increasing the scaling parameter, the increased cross-sectional area of the constructed rod should lead to greater stiffness as the second moment of area of the design is increased. On the other hand, the increase in the edge lengths towards or beyond a significant fraction of the persistence length of double-stranded DNA will transition the edges from beamlike mechanics to a soft-wire regime with uncorrelated ends. Consequently, one should observe a broad distribution on the end-to-end lengths of the dsDNA rendering of the edges, thereby reducing the observed persistence length of the assembled rods.

Here we use the observed persistence length as a measure of member/wireframe stiffness as it is easily calculable from the observed configurations in experiment and simulation; In general the persistence length of a structure, $p$, is related to its bending stiffness, $Y I$, through the expression $p=\frac{Y I}{k_{B} T}$ where $k_{B}$ is the Boltzmann constant and $T$ is temperature. ${ }^{32}$ For small edge lengths $l$ much below the persistence length of dsDNA, the dsDNA edges can be modeled as spring-like structural members, ${ }^{12,14}$ and thus one can calculate the expected increase in bending stiffness of a wireframe rod compared to the dsDNA member. For a beam-like wireframe rod 
with triangular cross section, increasing the cross-sectional area will serve to increase the bending stiffness and thus the persistence length according to formula $\mathrm{a}^{32,33}$

$$
\frac{p_{\text {frame }}}{p_{D N A}}=\frac{I_{\text {frame }}}{I_{D N A}}=\frac{2 l^{2}}{r_{\text {eff }}^{2}},
$$

where $p_{\text {frame }}$ and $p_{D N A}$ are the persistence lengths of the wireframe rod and the dsDNA member elements respectively, $I_{\text {frame }}$ and $I_{D N A}$ are the second moment of area of the wireframe rod and dsDNA member respectively, $l$ is the length of the transversal members in the wireframe (this expression is valid for the geometry generated by either isotropic scaling and base scaling, see Figure 1), and $r_{\text {eff }}$ is an effective structural radius of the dsDNA member elements that takes into account the interactions between other elements and the surrounding media.

A second mechanism is present within the wireframe rod design which, for high member lengths, will serve to decrease the persistence length of the rod: As the member elements within the wireframe rod achieve a significant fraction of their persistence length, increased variability in the end-to-end length of the members will become apparent. ${ }^{34}$ Consequently, the wireframe rod will no longer behave as a beam-like object but will rather be dominated by thermal fluctuations of its members. Given the distribution of end-to-end lengths of the dsDNA members, one can calculate the expected geometric form of the wireframe rod. As the member lengths reach a greater fraction of their persistence lengths, one will observe greater fluctuations of their configuration, and thus a decreasing persistence length of the wireframe rod.

These two effects can be estimated separately as shown in Figure 1. For both isotropic and base scaling, these two competing mechanisms lead to an increase in persistence length of the wireframe for small member lengths (where bending occurs on the length scale of the wireframe made up of multiple unit cells, and the member persistence length is thus dominated), and decreasing persistence length for large member lengths (where bending occurs on the length 
scale of the member elements); this analysis predicts that for some intermediate value, an optimal member length will exist. The value of $r_{\text {eff }}$ in Eq. (1) is chosen so that the value of $l / p_{D N A}$ corresponding to the peak value of $p_{\text {frame/ }} p_{D N A}$ matches the values found in the coarse grain molecular dynamics simulations detailed below. It is found that the value estimated for $r_{\text {eff }}$ in this way is $1.3 \mathrm{~nm}$, which is in close agreement with other work. ${ }^{35}$

For further in silico analysis of the wireframe DNA origami structures, we used oxDNA, a software package for coarse-grained molecular dynamics simulation of DNA. ${ }^{36,37}$ It was initially used to study the dynamics of small DNA systems but has evolved to a capacity to simulate large DNA nanostructures with thousands of nucleotides. This development was accelerated when the package started supporting GPU-based simulations, considerably decreasing the simulation time for large DNA systems. ${ }^{38}$

The simulation of DNA structures allows the study of small design modifications without extensive experimental work. It also allows us to vary the salt concentration in the simulation, a parameter that may have a large effect on the mechanics of DNA nanostructures. The salt concentration can also be varied in an experimental setting, but it is challenging to study this effect as the microscopy techniques commonly used rely on certain salt and buffer conditions for successful sample preparation.

To study the effect of salt concentration on the mechanics of DNA wireframe nanostructures, we simulated, in duplicate runs, a triangular-cross-section rod in several sodium ion concentrations between 100 and $735 \mathrm{mM}$. The persistence lengths were estimated at all concentrations and are plotted in Figure 2C. This study revealed a significant decrease in persistence length with increasing salt concentration. Increasing salt concentration from 100 to $500 \mathrm{mM}$ led to an $80 \%$ drop in persistence length. This effect was also apparent in a rod with a 
hexagonal cross-section (Supplementary Figure 1). Increasing salt concentration is known to reduce the persistence length of DNA. This effect, however, is largest at low salt concentrations ( $<20 \mathrm{mM}$ monovalent ions) and should be small in the salt concentration range studied here. ${ }^{39}$ We hypothesize that electrostatic repulsion within the structure acts as an inflating force, thus increasing the stiffness of the structure. When salt is introduced, it acts to reduce the electrostatic repulsion, hence decreasing the inflating force in the structure and consequently the stiffness of the structure. In the oxDNA model, ions are not modeled explicitly but instead incorporated as a screening of the charges based on the Debye-Hückel model. ${ }^{37}$ This in effect means that at lower salt concentrations the repulsive forces between DNA backbones will be stronger. The strength of these interactions have been parametrized to match experimental data on DNA.

To study the effect of isotropic scaling, we designed seven triangular-cross-section rods whose transversal edge-lengths range from 10 to $25 \mathrm{~nm}$. These structures were simulated in triplicate runs at $500 \mathrm{mM} \mathrm{Na}$; the resulting persistence lengths are plotted in Figure 2D. The uncertainty in the simulations appears to grow with the larger scaling values, making conclusions about optimal member lengths challenging. Additionally, the inflating effect of the electrostatic repulsion should be greatest for the small edge lengths, which adds a third effect not modeled in our beam theory analysis.

To study the effect of scaling only the cross-section size, we designed six triangular rods whose transversal edges have lengths between 10 and $23 \mathrm{~nm}$, with a fixed axial edge length of $10 \mathrm{~nm}$. These structures were simulated in duplicate runs at both 150 and $500 \mathrm{mM} \mathrm{Na}^{+}$. This revealed a clear peak behavior (Figure 2E) at a cross section side length of around $16 \mathrm{~nm}$, consistent with the beam theory analysis. This effect was visible in both salt concentrations, with the simulations at $150 \mathrm{mM} \mathrm{Na}^{+}$yielding consistently higher persistence length values. 
We also designed rods with varying side-count in the cross-section polygons, that is, rods with triangular, square, pentagonal and hexagonal cross-sections. These designs were simulated in duplicate runs at $500 \mathrm{mM} \mathrm{Na}^{+}$concentration. We observed a strong increase in persistence length with increasing number of sides in the cross-section polygon (Figure $2 \mathrm{~F}$ ).

The effect of the staple breakpoints on structural stability was simulated by removing, before simulation, all the breakpoints of a rod with triangular cross-section. This emulates the removal of staple breakpoints through experimental ligation, see below. We performed simulations in duplicate runs at $500 \mathrm{mM} \mathrm{Na}^{+}$and found that the removal of breakpoints greatly increases the persistence length of the assembled structures (Supplementary Figure 1). Literature indicates that nicks can reduce the persistence length of DNA, although this effect appears to decrease with increasing salt concentration. ${ }^{40}$

To validate the effects of the design choices experimentally, we designed six rods with varying cross-section profiles and sizes. This allowed us to study both the effect of the cross-section polygon type as well as the effect of scaling of the mesh when generating the DNA designs. The structures were assembled in phosphate buffered saline (PBS) that has a physiological concentration of sodium at around $150 \mathrm{mM}$. After folding, the structures were purified to remove excess staple strands and examined using negative stained TEM and atomic force microscopy (Figure 3). From the TEM images we traced the contours of the structures, and from this data estimated their persistence lengths.

To investigate the effect of isotropic scaling, we created three square-cross-section rods of three different side lengths: 13, 16 and $21 \mathrm{~nm}$. From the TEM data (Figure 3), it appears that the smallest scaling is significantly less rigid than the larger scalings and the $21 \mathrm{~nm}$ side length rod is slightly more rigid than the $16 \mathrm{~nm}$ side length rod. 
We also studied the effect of cross-section profile by comparing structures with similar edgelengths but different polygon types: triangular, square and hexagonal cross-sections. We found that the persistence length of the assembled structure increases with the number of sides in the cross-section polygon (Figure: 3A, 3C, 3E).

To study the effect of staple breakpoints, we assembled a triangular rod with staple strands modified to contain a $5^{\prime}$ phosphate group not normally found in synthetic DNA. This allowed us to remove the staple breakpoints by ligation using T4 ligase enzyme. We found that the persistence length of the structure nearly doubled after ligation (Figure 3F).

In addition, we tested if the structures could be reinforced by changing the wireframe members from single dsDNA edges to double dsDNA edges. For this purpose, we modified the BSCOR pipeline to allow for the introduction of double dsDNA renderings of desired edges. This was tested by the creation of a rod with a square cross-section where the axial members were constructed from double dsDNA edges and all other members were constructed from single dsDNA edges (Figure 3G). Note that these double dsDNA edges were only attached by crossover at the vertices, i.e. no internal junctions in the members were introduced. We found that this reinforced rod has a persistence length that was about $60 \%$ greater than the corresponding rod without reinforcement while using only about $30 \%$ more material (Figure $3 G)$.

To investigate the stiffness of wireframe DNA origami structures on a larger scale, we designed two rods, one with a square and the other with an octagonal cross-section, that are capable of polymerizing to long wires. After folding, the rods were imaged in TEM and had assembled to wires several micrometers long, with the octagonal cross-section wire showing a persistence length of approximately $6 \mu \mathrm{m}$ (Figure 4). 
In addition to negative stained TEM where the structures are imaged in a dry state, we also performed cryo-electron microscopy on the rod with hexagonal cross-section (Supplementary Figure 2). Interestingly, the persistence length measured from this imaging was more than twice as large as the measurement in negative stained TEM. This may indicate that the dry state of negative stained TEM deforms the wireframe structures, giving them a lower apparent persistence length. Previous studies indicate that DNA origami assembly may be imperfect, and that a typical structure may lack some staple strands. ${ }^{41,42}$ This may be a contributing source to the quantitative difference between the persistence length values measured in simulation where the simulated structures are perfectly assembled, and in experiments where the exact staple incorporation yield is unknown. We believe that tolerance to imperfect assembly should be a design consideration and an interesting area for further study.

\section{Conclusions}

Bringing the benefits of wireframe construction to nanoscale DNA devices is an attractive proposition, especially as the sparser designs lead to less stringent constraints on salt concentrations, allowing for assembly and stability in physiological buffers. The optimal design choices for wireframe DNA nanostructures are not necessarily the same as in macroscopic latticework assemblies, particularly due to significant contributions from thermal fluctuations at the nanoscale. Here we use a combination of structural mechanics, molecular dynamics simulations and experiments to study the effect of various parameters on the stiffness of wireframe DNA origami structures. 
We find that the effect of edge-length is non-trivial: In both the isotropic and base scaling regimes, our theoretical analysis predicts that an optimal edge-length exists. Though this optimal edge length is not observed in coarse-grain molecular dynamics simulations of the isotropic scaling data set, it is clearly visible in the base scaling data for both salt concentrations investigated. The sensitivity of the simulation results to the environment (including, for example, salt concentration which is not considered in the theory work) make quantitative agreement currently challenging. Increasing the side-count in the cross-section polygon of a wireframe rod greatly increases persistence length in both simulations and experiments, although this increase comes at a cost of more material use per unit length of the wireframe rod. Experiments and simulations reveal that the staple break points positioned on each edge appear to reduce the stiffness of the assemblies. Staple strand ligation in the assembled structure increases stiffness and possibly also reduces exonuclease activity. Simulations also reveal that structural stiffness is greatly reduced with increasing concentration of monovalent salts. The findings in this study can be used as a guide for the design of wireframe DNA nanostructures with optimal stiffness.

\section{Methods/Experimental}

\section{Structure design}

All structures synthesized in this study were designed using vHelix and the BSCOR package, which are tools to generate DNA origami designs from polyhedral meshes. The initial polyhedral mesh models were generated in Autodesk Maya and exported in the STL format. These were then converted to the ASCII PLY format using the software MeshLab. After conversion, the BSCOR package was used to generate scaffold routings through the mesh wireframes and to convert the wireframes to coarse-grained DNA geometries using rigid-body-model simulations. 
The BSCOR package generated the initial DNA designs in the RPOLY format that were then imported to Autodesk Maya running the DNA design plugin vHelix. In vHelix, the strains of the structures were further relaxed by the introduction of unpaired bases in some vertices using the feature 'auto fill strand gaps'. Next, the scaffold strands were assigned their sequences, thereby automatically generating the sequences of the complementary staple strands. The staple strand sequences were further exported and processed in Microsoft Excel. Unpaired nucleotides in the staple strands, whenever present, were assigned as adenines.

\section{Assembly of structures}

Staple oligonucleotides were purchased from Integrated DNA Technologies. The scaffold strand was prepared from M13 phage variants as described before. ${ }^{28}$ Folding reactions were prepared with a scaffold strand concentration of $5 \mathrm{nM}$ and staple strand concentrations of $50 \mathrm{nM}$ each in either 1x PBS (Sigma Aldrich) or $10 \mathrm{mM} \mathrm{MgCl}_{2}$ (Sigma Aldrich), $5 \mathrm{mM}$ tris (VWR) and $1 \mathrm{mM}$ EDTA (VWR). The folding reactions were put in a thermocycler on an annealing ramp starting with a heat denaturation at $80{ }^{\circ} \mathrm{C}$ for 5 min followed by cooling from $80{ }^{\circ} \mathrm{C}$ to $60{ }^{\circ} \mathrm{C}$ over $20 \mathrm{~min}$, then a slow cooling from $60{ }^{\circ} \mathrm{C}$ to $24^{\circ} \mathrm{C}$ over $14 \mathrm{~h}$.

After folding, ultrafiltration was used to remove excess staples from the folding reactions. The samples were transferred to amicon $100 \mathrm{~K}$ filters (Millipore) and diluted to $500 \mu \mathrm{l}$ in folding buffer. The filters were centrifuged at $14000 \mathrm{x}$ g for 2 minutes and the flow through was discarded. The sample in the filter was again diluted to $500 \mu 1$ in the folding buffer and centrifuged at $14000 \mathrm{x} \mathrm{g}$ for 2 minutes. The sample in the filter was diluted to $100 \mu \mathrm{l}$ and used to repeatedly wash the filter walls before inverting the filter in an empty tube and centrifuging it at $1000 \mathrm{x} \mathrm{g}$ for 2 minutes.

\section{AFM}


For AFM imaging, samples folded in magnesium buffer were used. The samples were diluted 10 times in the folding buffer, and from this, $10 \mu 1$ was applied to freshly cleaved mica. After 30 seconds of incubation under a petri dish, $4 \mu \mathrm{l}$ of $5 \mathrm{mM} \mathrm{NiSO}_{4}$ (VWR) was added and the sample was incubated for further 4.5 minutes. After this, the mica surface was washed by applying $1 \mathrm{ml}$ of folding buffer and then removing it using a pipette. After washing, $1.5 \mathrm{ml}$ of folding buffer was added to the sample for imaging.

Imaging was performed using a JPK instruments nanowizard 3 ultra with an Olympus Biolever mini cantilever in AC mode.

\section{TEM}

For TEM experiments, samples folded in PBS were used. Uranyl formate stain (EMS diasum) was prepared in $400 \mu \mathrm{l}$ aliquots at $2 \% \mathrm{w} / \mathrm{v}$. An $8 \mu \mathrm{l}$ of $1 \mathrm{M} \mathrm{NaOH}$ was added to one aliquot that was then centrifuged at $16,500 \mathrm{x}$ g for 5 minutes. Formvar carbon grids (EMS diasum) were glow discharged for 20 seconds and then spotted with $3 \mu 1$ of sample for 20 seconds. The grid was then spotted on a filter paper and then on a $50 \mu \mathrm{l}$ drop of $\mathrm{H} 20$ for 3 seconds. After another spotting on the filter paper, the grid was put on a $50 \mu 1$ drop of the uranyl formate stain for 20 seconds. The grid was again spotted on the filter paper and allowed to air dry and then imaged in a FEI Morgagni 268 at $22000 \times$ magnification.

\section{Coarse grained simulations}

The vHelix design files were saved in the Maya ASCII (MA) format and converted to the oxDNA format using a converter developed at the Doye group in Oxford. These design files may contain non-physical geometry, potentially leading to extreme artificial backbone forces 
which cause simulation failure. This was addressed by relaxing the geometries in two precursor simulations where the backbone forces were set to a maximal possible value. After this, the structures were simulated for $10^{8}$ time-steps, which corresponds to approximately $1.5 \mu \mathrm{s}$ simulation time. Simulations were performed with an Anderson-like thermostat at $30{ }^{\circ} \mathrm{C}$ using the oxDNA2 model. Snapshots of simulation trajectories were saved every 20000 time steps, resulting in 5000 trajectory data points per simulation.

\section{Calculation of persistence length from TEM data.}

For each rod, TEM images of at least 100 structures were collected. A custom Python script was used to calculate persistence length from the TEM data. For each imaged structure, a spine was created through the structure by positioning points spaced $11 \mathrm{~nm}$ apart along the trajectory. The correlation between tangent vectors separated a distance $l$ along the trajectory of a structure is expected to decay according to:

$$
\left\langle\overrightarrow{t_{1}} \cdot \overrightarrow{t_{2}}\right\rangle=e^{-\frac{l}{s p}}
$$

Where $p$ is the persistence length of the structure and $s$ is a surface parameter set to 2 for $2 \mathrm{D}$ images of nanostructures. ${ }^{43}$

In the software, tangent vectors were created between adjacent points along the spine of the structure, and then cosines of the angles between vector pairs were calculated for vector pairs of increasing separation along the spine. To this data, the above function was fitted to estimate the persistence length $p$.

\section{Calculation of persistence length from simulation data.}

In the simulated trajectories, the first 500 data-points corresponding to the first $10^{7}$ simulation steps were discarded to remove influence from the initial conformation. 
In each structure, a nucleotide was selected in the middle of each edge of each cross-section plane. The coordinates of these nucleotides were then used to calculate the center point of each cross-section plane in a given time-step. These coordinates were extracted from the simulation trajectories in all time steps and the center point of each cross-section plane was calculated, yielding a $3 \mathrm{D}$ spine for each time point. Tangent vectors to the spine were created between adjacent center points. The cosines of the angles between these tangent vectors were calculated for vectors separated by increasing path length $l$ along the spine. Again, equation (2) was used to fit the persistence length to the calculated values but with the surface parameter $s$ set to 1 for $3 \mathrm{D}$ data.

\section{Theory prediction}

For a given length $s$ of dsDNA, the realized end-to-end length follows a probability distribution $p(s) .{ }^{34}$ Due to this uncertainty in end-to-end length, for a given wireframe unit cell of edge length $l$, the realized geometry will deviate from its designed ideal. To calculate the realized geometry of a wireframe unit cell of ideal edge length $l$, and thereby estimate the wireframe rod's observed persistence length, we randomly take values from $p(s)$, with $s=l$, assigning these end-to-end lengths to the edges of the wireframe unit cell. Given these realized edge lengths we calculate the positions of the nodes of the wireframe unit cell; we note that as the wireframe is isostatic, ${ }^{44}$ the node positions are well defined without encountering states of self-stress. The orientation of the upper plane relative to the lower plane of the structure is then calculated (see Figure 1) which allows us to estimate the observed persistence length of the structure. $^{45}$ 
The persistence length due to beam-like behavior is calculated assuming the dsDNA members behave as beams with constant circular cross-section of radius $r_{\text {eff }}$ made of an isotropic material. The second moment of area of this dsDNA can thus be estimated as,

$$
I_{D N A}=\frac{\pi r_{e f f}^{4}}{4} .
$$

The second moment of area of a wireframe with triangular cross section, with constant member length $l$, made from circular beams of radius $r$ eff, can be calculated as

$$
I_{\text {frame }}=\frac{\pi r_{e f f}^{2} l^{2}}{2} .
$$

Assuming an isotropic construction material, the increase in persistence length of the wireframe with respect to the dsDNA members can thus be calculated using the expressions given in the main text, resulting in Eq. 1. 
A.
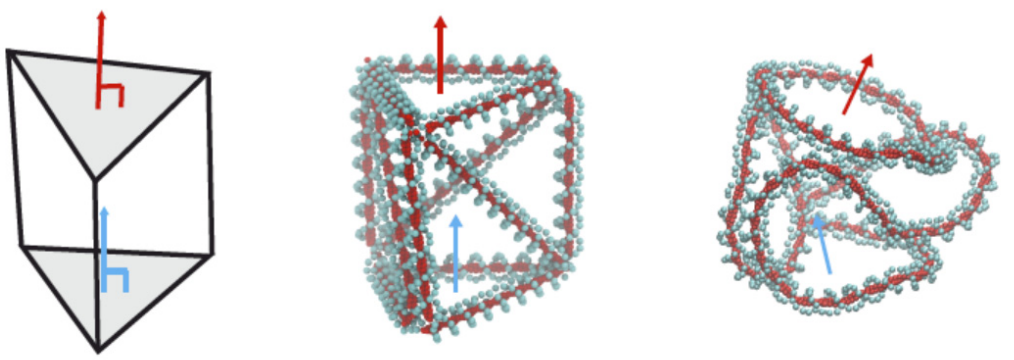

B.

Isotropic scaling
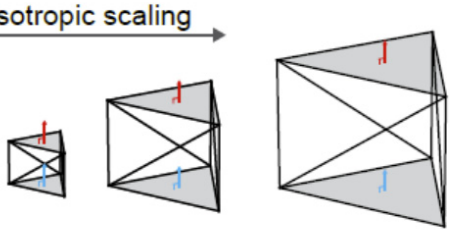

Base scaling
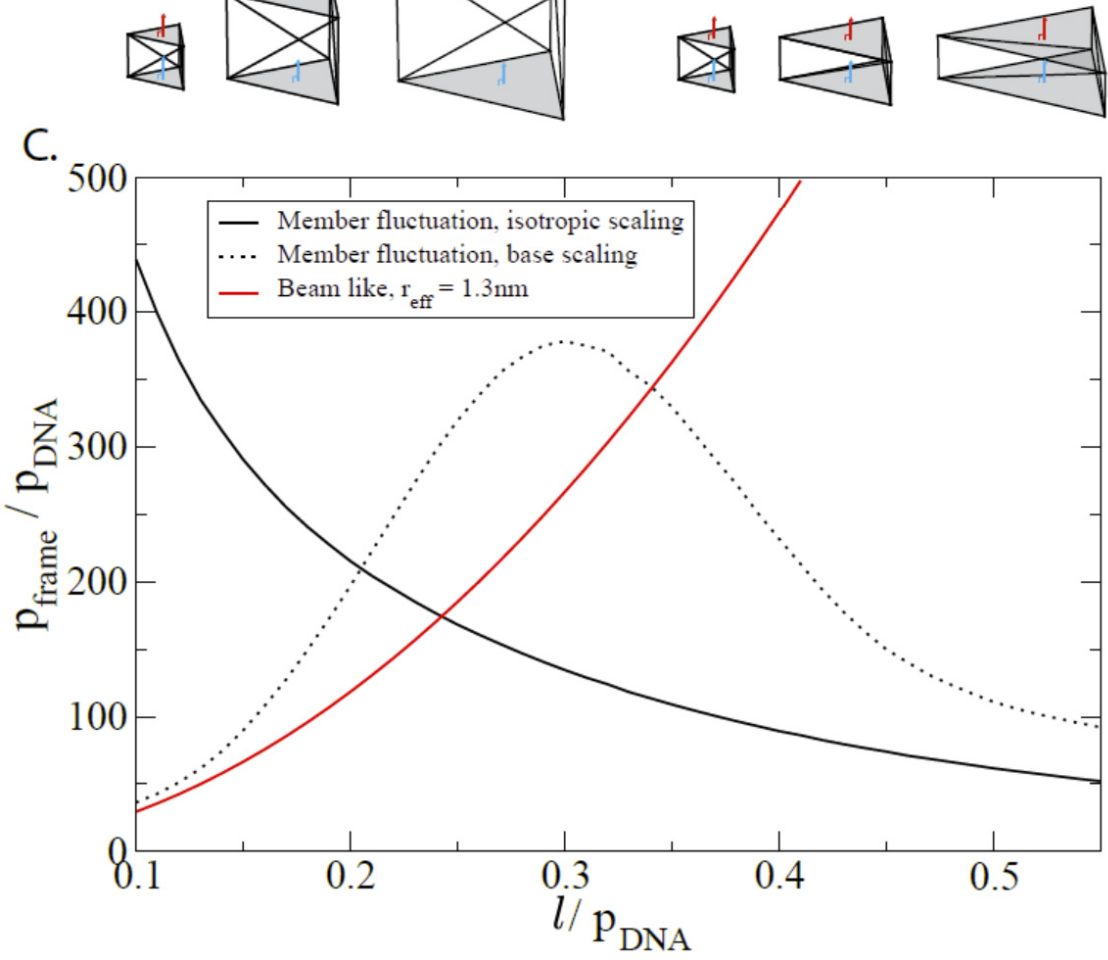

Figure 1: The effects of isotropic and base scaling as predicted by theory. (A) In an idealized wireframe unit cell the bottom and top planes are parallel (left and center). In a realistic DNA representation, thermal fluctuations will lead to non-parallel planes (right). (B) As the scaling of the structure increases, thermal fluctuations of the edge lengths will increase. We study this phenomenon for isotropic scaling, where all edges increase in length uniformly, and for base scaling, where the length of the axial edges remains constant as the transversal edges scale up. 
(C) For small member length $(l)$, the beam-like behavior dominates, and thus increasing the member length serves to increase the persistence length of the wireframe rods. For large member length, thermal fluctuations of members dominate wireframe behavior and further increases in member length decrease the persistence length. For both scalings, an optimal member length is observed. In both cases, the effective structural radius of the dsDNA members is taken to be 1.3 nm. The results shown are independent of the persistence length of the dsDNA members (including variations due to temperature ${ }^{35}$ or environment ${ }^{39}$ ) or alternative construction materials (where $p_{D N A}$ would take the value of the persistence length of the construction members used). 
A.

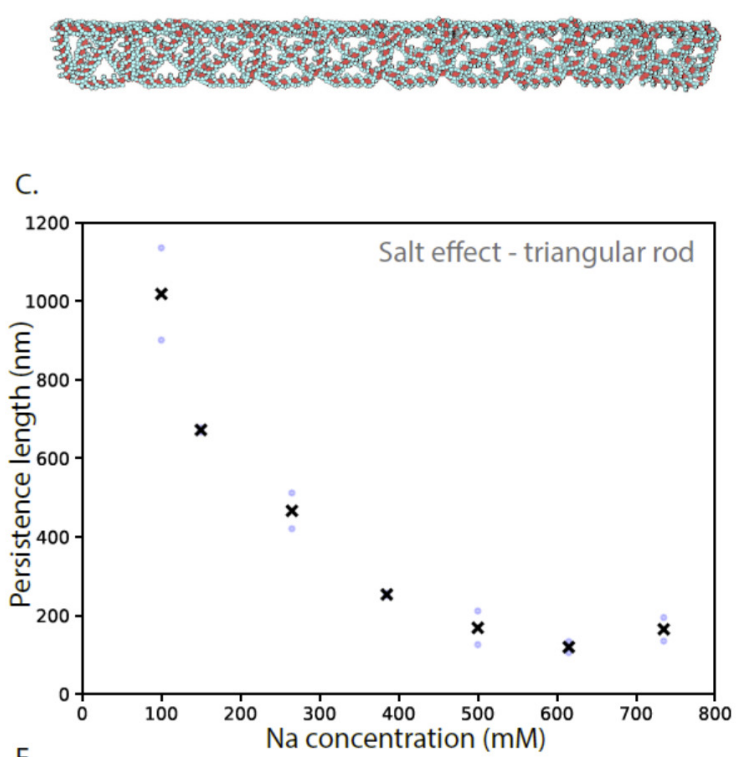

E.

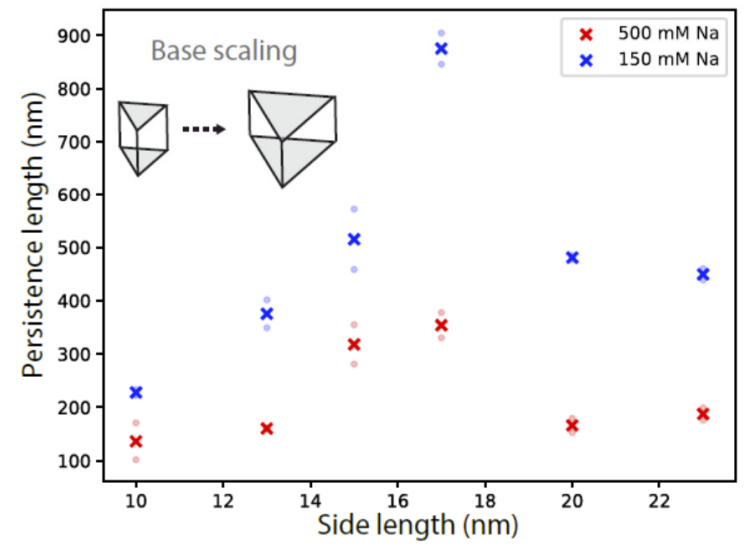

B.
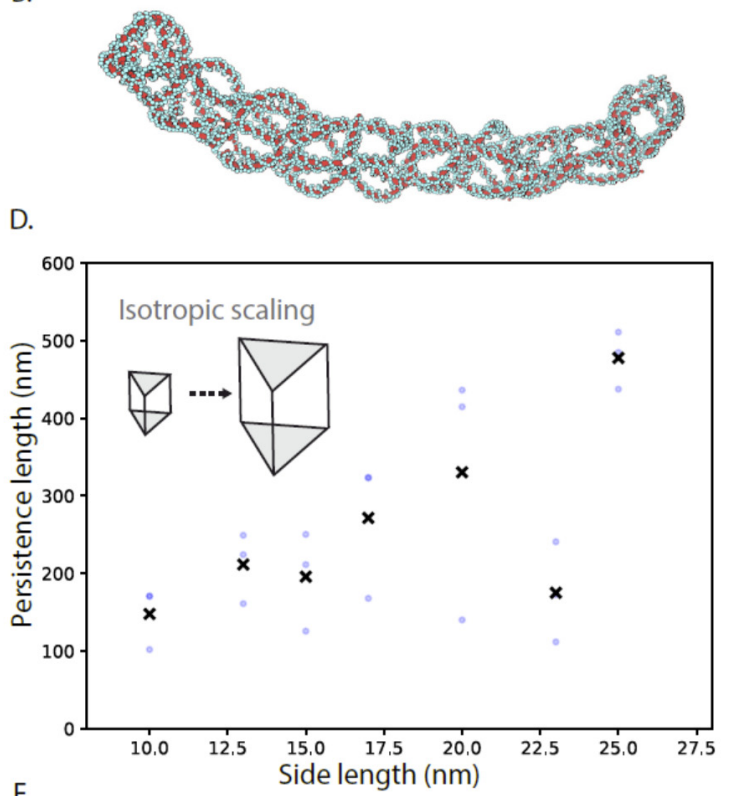

F.

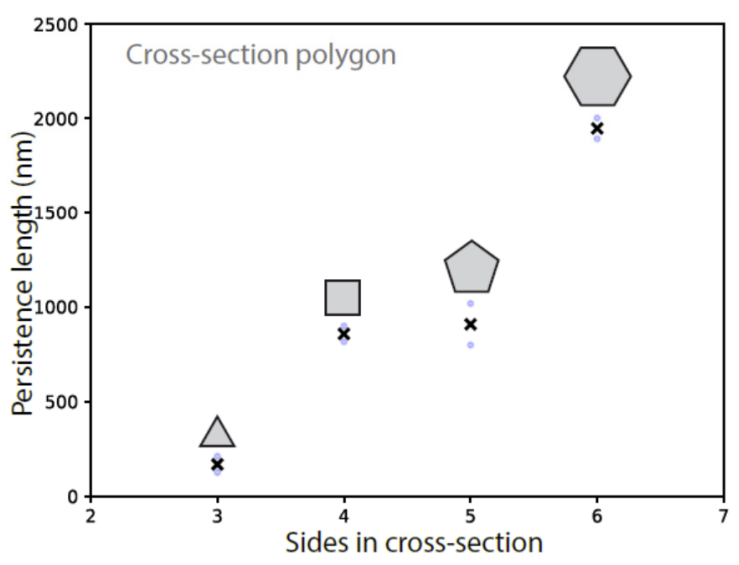

Figure 2. Persistence lengths of various designs (D)-(F), as well as one design under different salt conditions (C), calculated from oxDNA simulations. (A) Rendering of a rod with a triangular cross-section before and (B) after an oxDNA simulation. (C) A triangular rod with edge lengths of $15 \mathrm{~nm}$ simulated in various sodium ion concentrations. (D) Simulations of isotropicallyscaled triangular rods at $500 \mathrm{mM} \mathrm{Na}^{+}$concentration. (E) Simulation of triangular rods with axial 
edge lengths fixed to $10 \mathrm{~nm}$ but with the transversal/base edge lengths varied. Simulations were performed at both $150 \mathrm{mM}$ and $500 \mathrm{mM} \mathrm{Na}^{+}$. (F) Simulations of rods made from ten units, each with edge lengths of around $15 \mathrm{~nm}$ but with varying type of cross-section polygons. An x marker shows average value and the circle markers indicate values of individual replicate simulations.

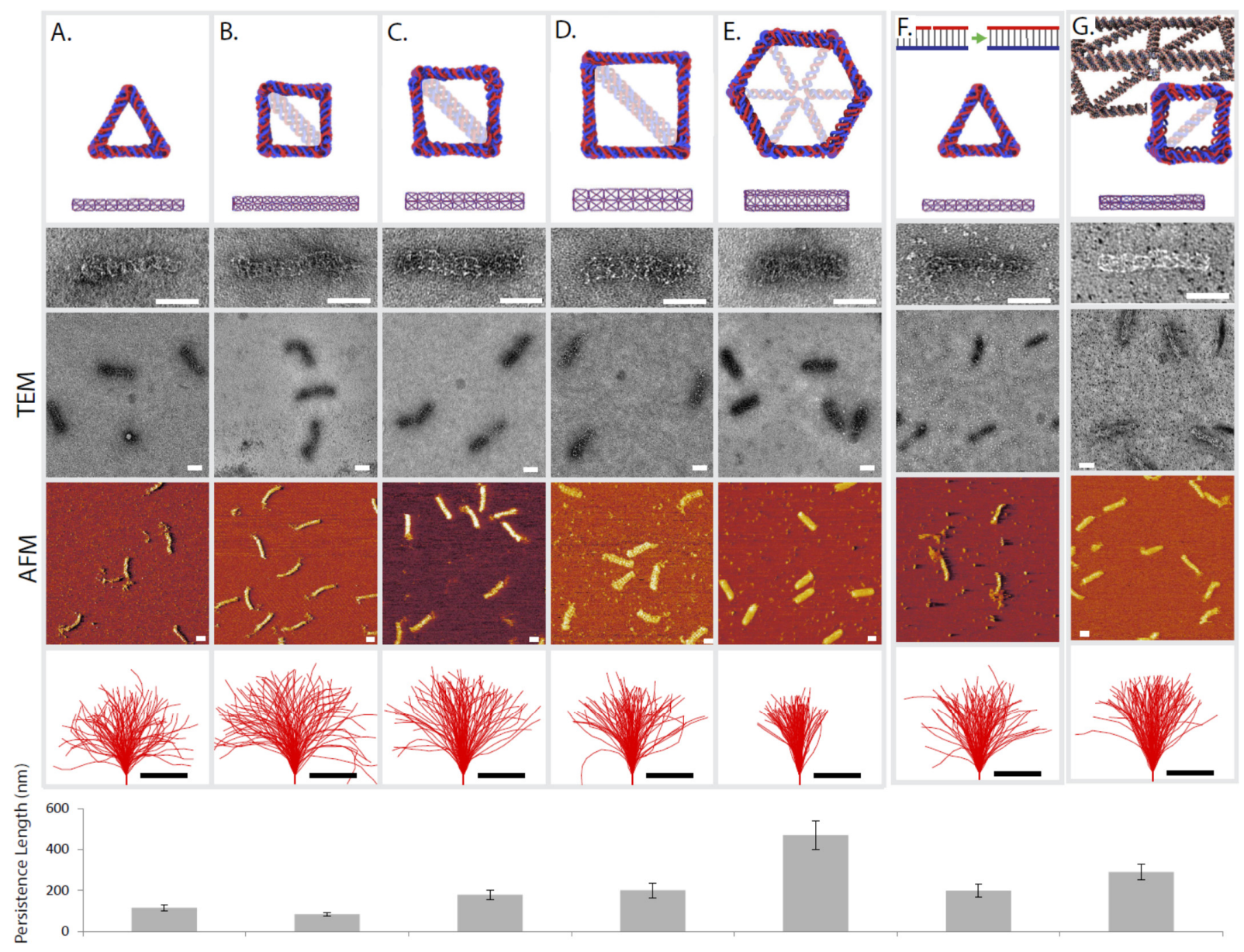

Figure 3. Wireframe DNA origami rods designed with varying cross section polygons and scaling values. Top: front and side rendering of vHelix designs of rods, from left: (A) a rod with a triangular cross-section and edge length $15 \mathrm{~nm},(\mathrm{~B})$-(D) three isotropically scaled rods with a square cross-section and edge lengths of 13,16, $21 \mathrm{~nm},(\mathrm{E})$ a rod with a hexagonal cross-section, (F) a triangular rod with edge length $15 \mathrm{~nm}$ and staple breakpoints removed by ligation, $(\mathrm{G}) \mathrm{a}$ 
square rod with edge length $15 \mathrm{~nm}$ and axial edges rendered by double DNA duplexes. All structures are hollow, the visible cross-bars are only on the top and bottom caps, and are grayed out to signify this. Second and third row: TEM images of folded structures. Fourth row: AFM images of folded structures. Fifth row: Contour trajectories of 100 structures of each type, extracted from TEM images. Bottom row, persistence lengths calculated from TEM data. All scale bars are $50 \mathrm{~nm}$.

A.

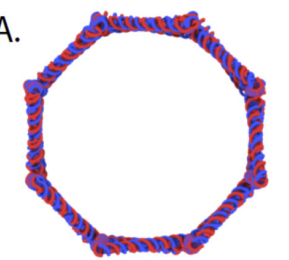

B.
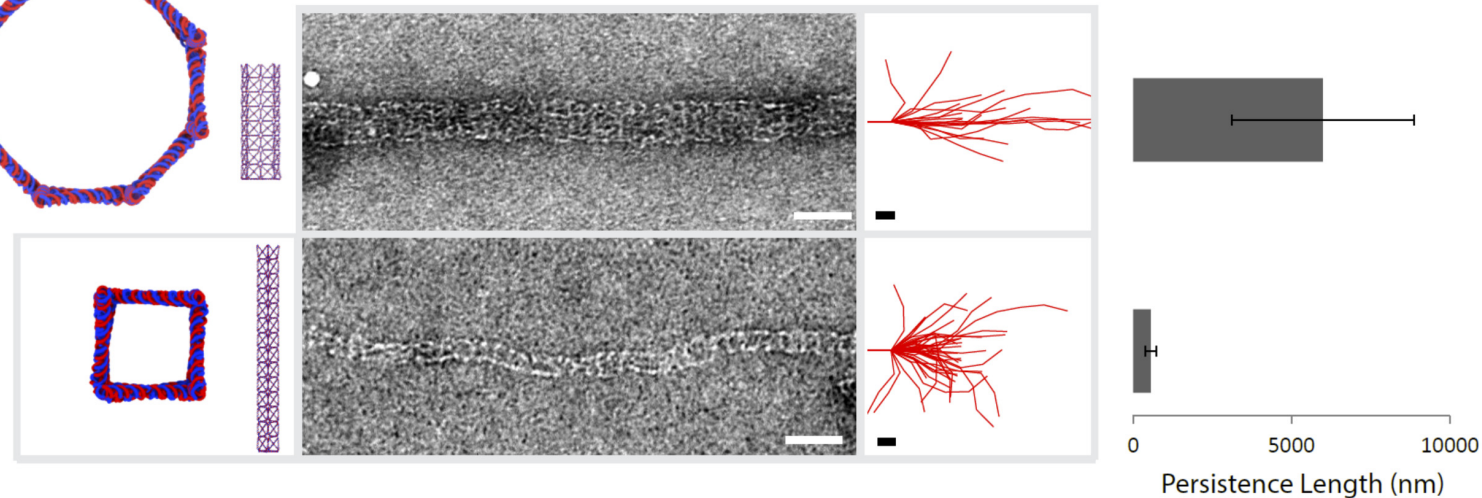

Figure 4. Wireframe DNA origami tubes with octagonal (A) and square (B) cross-sections, and edge lengths of $16 \mathrm{~nm}$, were designed with linkers to polymerize into multimer wires. Left: front and side renders of the monomer units. Second column: TEM images of folded and assembled multimer wires. Third column: trajectories of multimer wires extracted from TEM images. Fourth column: persistence length values calculated from TEM data for the square and octagonal cross-section wires. All scale bars $50 \mathrm{~nm}$. 


\section{Supporting Information.}

Supplementary materials (PDF)

\section{AUTHOR INFORMATION}

\section{Corresponding Authors}

*pekka.orponen@aalto.fi

*bjorn.hogberg@ki.se

\section{ACKNOWLEDGMENT}

This work was funded through grants from the Swedish Foundation for Strategic Research (grant FFL12-0219 to B.H.), the ERC (grant 724872 to B.H.) and the Knut and Alice Wallenberg foundation (Academy Fellow grant KAW2014.0241 to B.H.). The work of A.M. and P.O. has been supported by Academy of Finland grant 311639, "Algorithmic Designs for Biomolecular Nanostructures (ALBION)". In addition, A.M. has been funded by the Helsinki Doctoral Education Network on Information and Communications Technology (HICT) and by a Nokia Foundation scholarship. We further wish to acknowledge the computational resources provided by the Science-IT project of Aalto University, and by CSC - the IT Center for Science, Finland. The cryo-EM data was collected at the Cryo-EM Swedish National Facility funded by the Knut and Alice Wallenberg, Family Erling Persson and Kempe Foundations, SciLifeLab, Stockholm University and Umeå University. D.R.-K. acknowledges funding from the Academy of Finland postdoctoral grant 13287859. We would like to thank Domen Prešern and Jonathan Doye for assistance with oxDNA simulations.

\section{SUPPORTING INFORMATION}

Additional simulations of wireframe structures (Supplementary Figure 1), Cryo TEM data of rod with hexagonal cross-section (Supplementary Figure 2), Additional TEM data of wireframe rods (Supplementary Figures 3-10). 


\section{References}

(1) Seeman, N. C. Nucleic Acid Junctions and Lattices. J. Theor. Biol. 1982, 99, 237-247.

(2) Chen, J. H.; Seeman, N. C. Synthesis from DNA of a Molecule with the Connectivity of a Cube. Nature 1991, 350, 631-633.

(3) Rothemund, P. W. K. Folding DNA to Create Nanoscale Shapes and Patterns. Nature 2006, 440, 297-302.

(4) Kuzyk, A.; Schreiber, R.; Fan, Z.; Pardatscher, G.; Roller, E.-M.; Högele, A.; Simmel, F.

C.; Govorov, A. O.; Liedl, T. DNA-Based Self-Assembly of Chiral Plasmonic Nanostructures with Tailored Optical Response. Nature 2012, 483, 311-314.

(5) Gopinath, A.; Miyazono, E.; Faraon, A.; Rothemund, P. W. K. Engineering and Mapping Nanocavity Emission via Precision Placement of DNA Origami. Nature 2016, 535, 401405.

(6) Maune, H. T.; Han, S.-P.; Barish, R. D.; Bockrath, M.; Iii, W. A. G.; Rothemund, P. W. K.; Winfree, E. Self-Assembly of Carbon Nanotubes into Two-Dimensional Geometries Using DNA Origami Templates. Nat. Nanotechnol. 2010, 5, 61-66.

(7) Jin, Z.; Sun, W.; Ke, Y.; Shih, C.-J.; Paulus, G. L. C.; Hua Wang, Q.; Mu, B.; Yin, P.; Strano, M. S. Metallized DNA Nanolithography for Encoding and Transferring Spatial Information for Graphene Patterning. Nat. Commun. 2013, 4, 1663.

(8) Shaw, A.; Lundin, V.; Petrova, E.; Fördős, F.; Benson, E.; Al-Amin, A.; Herland, A.; 
Blokzijl, A.; Högberg, B.; Teixeira, A. I. Spatial Control of Membrane Receptor Function Using Ligand Nanocalipers. Nat. Methods 2014, 11, 841-816.

(9) Douglas, S. M.; Bachelet, I.; Church, G. M. A Logic-Gated Nanorobot for Targeted Transport of Molecular Payloads. Science 2012, 335, 831-834.

(10) Douglas, S. M.; Dietz, H.; Liedl, T.; Högberg, B.; Graf, F.; Shih, W. M. Self-Assembly of DNA into Nanoscale Three-Dimensional Shapes. Nature 2009, 459, 414-418.

(11) Han, D.; Pal, S.; Nangreave, J.; Deng, Z.; Liu, Y.; Yan, H. DNA Origami with Complex Curvatures in Three-Dimensional Space. Science 2011, 332, 342-346.

(12) Schiffels, D.; Liedl, T.; Fygenson, D. K. Nanoscale Structure and Microscale Stiffness of DNA Nanotubes. ACS Nano 2013, 7, 6700-6710.

(13) Pfitzner, E.; Wachauf, C.; Kilchherr, F.; Pelz, B.; Shih, W. M.; Rief, M.; Dietz, H. Rigid DNA Beams for High-Resolution Single-Molecule Mechanics. Angew. Chemie Int. Ed. 2013, 52, 7766-7771.

(14) Simmel, S. S.; Nickels, P. C.; Liedl, T. Wireframe and Tensegrity DNA Nanostructures. Acc. Chem. Res. 2014, 47, 1691-1699.

(15) Douglas, S. M.; Marblestone, A. H.; Teerapittayanon, S.; Vazquez, A.; Church, G. M.; Shih, W. M. Rapid Prototyping of 3D DNA-Origami Shapes with CaDNAno. Nucleic Acids Res. 2009, 37, 5001-5006.

(16) Yoo, J.; Aksimentiev, A. In Situ Structure and Dynamics of DNA Origami Determined through Molecular Dynamics Simulations. Proc. Natl. Acad. Sci. U. S. A. 2013, 110, 20099-20104. 
(17) Doye, J. P. K.; Ouldridge, T. E.; Louis, A. A.; Romano, F.; Šulc, P.; Matek, C.; Snodin, B. E. K.; Rovigatti, L.; Schreck, J. S.; Harrison, R. M.; Smith, W. P. J.; Michon, A.; Osman, R.; Orozco, M.; Perez, A.; Singh, T.; Spackova, N.; Šponer, J. Coarse-Graining DNA for Simulations of DNA Nanotechnology. Phys. Chem. Chem. Phys. 2013, 15, 20395-20414.

(18) Zhang, Y.; Seeman, N. C. Construction of a DNA-Truncated Octahedron. J. Am. Chem. Soc. 1994, 116, 1661-1669.

(19) Shih, W. M.; Quispe, J. D.; Joyce, G. F. A 1.7-Kilobase Single-Stranded DNA That Folds into a Nanoscale Octahedron. Nature 2004, 427, 618-621.

(20) Goodman, R. P.; Schaap, I. A. T.; Tardin, C. F.; Erben, C. M.; Berry, R. M.; Schmidt, C. F.; Turberfield, A. J. Rapid Chiral Assembly of Rigid DNA Building Blocks for Molecular Nanofabrication. Science 2005, 310, 1661-1665.

(21) He, Y.; Ye, T.; Su, M.; Zhang, C.; Ribbe, A. E.; Jiang, W.; Mao, C. Hierarchical SelfAssembly of DNA into Symmetric Supramolecular Polyhedra. Nature 2008, 452, 198201.

(22) Han, D.; Pal, S.; Yang, Y.; Jiang, S.; Nangreave, J.; Liu, Y.; Yan, H. DNA Gridiron Nanostructures Based on Four-Arm Junctions. Science 2013, 339, 1412-1415.

(23) Inuma, R.; Ke, Y.; Jungmann, R.; Schlichthaerle, T.; Woehrstein, J. B.; Yin, P. Polyhedra Self-Assembled from DNA Tripods and Characterized with 3D DNA-PAINT. Science 2014, 344, 65-69.

(24) Zhang, F.; Jiang, S.; Wu, S.; Li, Y.; Mao, C.; Liu, Y.; Yan, H. Complex Wireframe DNA Origami Nanostructures with Multi-Arm Junction Vertices. Nat. Nanotechnol. 2015, 10, 
$779-784$.

(25) Benson, E.; Mohammed, A.; Bosco, A.; Teixeira, A. I.; Orponen, P.; Högberg, B. Computer-Aided Production of Scaffolded DNA Nanostructures from Flat Sheet Meshes. Angew. Chemie Int. Ed. 2016, 55, 8869-8872.

(26) Rayneau-Kirkhope, D.; Mao, Y.; Farr, R. Ultralight Fractal Structures from Hollow Tubes. Phys. Rev. Lett. 2012, 109, 204301.

(27) Gordon, J. E. Structures; Da Capo Press, 1991.

(28) Benson, E.; Mohammed, A.; Gardell, J.; Masich, S.; Czeizler, E.; Orponen, P.; Högberg, B. DNA Rendering of Polyhedral Meshes at the Nanoscale. Nature 2015, 523, 441-444.

(29) Veneziano, R.; Ratanalert, S.; Zhang, K.; Zhang, F.; Yan, H.; Chiu, W.; Bathe, M. Designer Nanoscale DNA Assemblies Programmed from the Top Down. Science (80-. ). 2016, 352, 1534.

(30) Matthies, M.; Agarwal, N. P.; Schmidt, T. L. Design and Synthesis of Triangulated DNA Origami Trusses. Nano Lett. 2016, 16, 2108-2113.

(31) Howard, J. Mechanics of Motor Proteins and the Cytoskeleton; Sinauer Associates, 2001.

(32) Landau, L. D.; Lifshitz, E. M. Theory of Elasticity; Pergamon Press, 1959.

(33) Timoshenko, S. P.; Gere, J. M. Theory of Elastic Stability,; McGraw Hill, 1986.

(34) Wilhelm, J.; Frey, E. Radial Distribution Function of Semiflexible Polymers. Phys. Rev. Lett. 1996, 77, 2581-2584. 
(35) Lu, Y.; Weers, B.; Stellwagen, N. C. DNA Persistence Length Revisited. Biopolymers 2001, 61, 261-275.

(36) Ouldridge, T. E.; Louis, A. A.; Doye, J. P. K. Structural, Mechanical, and Thermodynamic Properties of a Coarse-Grained DNA Model. J. Chem. Phys. 2011, 134.

(37) Snodin, B. E. K.; Randisi, F.; Mosayebi, M.; Šulc, P.; Schreck, J. S.; Romano, F.; Ouldridge, T. E.; Tsukanov, R.; Nir, E.; Louis, A. A.; Doye, J. P. K. Introducing Improved Structural Properties and Salt Dependence into a Coarse-Grained Model of DNA. J. Chem. Phys. 2015, 142, 234901.

(38) Rovigatti, L.; Šulc, P.; Reguly, I. Z.; Romano, F. A Comparison between Parallelization Approaches in Molecular Dynamics Simulations on GPUs. J. Comput. Chem. 2015, 36, 18.

(39) Baumann, C. G.; Smith, S. B.; Bloomfield, V. A.; Bustamante, C. Ionic Effects on the Elasticity of Single DNA Molecules. Proc. Natl. Acad. Sci. 1997, 94, 6185-6190.

(40) Furrer, P.; Bednar, J.; Stasiak, A. Z.; Katritch, V.; Michoud, D.; Stasiak, A.; Dubochet, J. Opposite Effect of Counterions on the Persistence Length of Nicked and Non-Nicked DNA. J. Mol. Biol. 1997, 266, 711-721.

(41) Myhrvold, C.; Baym, M.; Hanikel, N.; Ong, L. L.; Gootenberg, J. S.; Yin, P. Barcode Extension for Analysis and Reconstruction of Structures. Nat. Commun. 2017, 8, 1-9.

(42) Strauss, M. T.; Schueder, F.; Haas, D.; Nickels, P. C.; Jungmann, R. Quantifying Absolute Addressability in DNA Origami with Molecular Resolution. Nat. Commun. 2018, 9, 1-7.

(43) Lamour, G.; Kirkegaard, J. B.; Li, H.; Knowles, T. P. J.; Gsponer, J. Easyworm: An Open- 
Source Software Tool to Determine the Mechanical Properties of Worm-like Chains. Source Code Biol. Med. 2014, 9, 1-6.

(44) Meeussen, A. S.. Topological Principles for the Design of the Mechanical Metamaterials. Masters thesis, Leiden University, The Netherlands, 2016-03-31

(45) Schellman, J. A. Flexibility of DNA. Biopolymers 1974, 13, 217-226. 